\title{
Regulation of community functional composition across taxonomic variation by resource-consumer dynamics
}

\author{
Lee Worden
}

October 8, 2019

\begin{abstract}
High-throughput sequencing techniques such as metagenomic and metatranscriptomic technologies allow cataloging of functional characteristics of microbial community members as well as their taxonomic identity. Such studies have found that a community's composition in terms of ecologically relevant functional traits or guilds can be conserved more strictly across varying settings than taxonomic composition is. I use a standard ecological resource-consumer model to examine the dynamics of traits relevant to resource consumption, and analyze determinants of functional composition. This model demonstrates that interaction with essential resources can regulate the community-wide abundance of ecologically relevant traits, keeping them at consistent levels despite large changes in the abundances of the species housing those traits in response to changes in the environment, and across variation between communities in species composition. Functional composition is shown to be able to track differences in environmental conditions faithfully across differences in community composition. Mathematical conditions on consumers' vital rates and functional responses sufficient to produce conservation of functional community structure across taxonomic differences are presented.
\end{abstract}

\section{Introduction}

Microbes play a key role in every ecological community on earth, and are crucial to the health of plants and animals both as mutualists and as pathogens. Understanding the ecological function and dynamics of microbes is important to human health and to the health of the planet. Because microbes exhibit short generation times, rapid evolution, horizontal transmission of genes, and great diversity, and can coexist in a massive number of partially isolated local communities, the study of their communities can bring different 
questions to the fore than are raised in the more common traditions of ecological theory focused on plants and animals. Newly available techniques of high-throughput genetic and transcriptomic sequencing are making microbial community structure visible in detail for the first time.

One pattern appearing in microbial communities, in multiple very different settings, is that communities composed very differently in terms of species, genera, and even higher-level classifications of microbes can have much more similar structure when viewed in terms of the functional genes and genetic pathways present in the communities than when a catalog of taxa is constructed. Additionally, environmental changes can induce consistent changes in community-level abundance of relevant genes or pathways while leaving others unchanged, in situations where such a pattern is not readily visible in taxonomic data due to high taxonomic variability across communities. Metagenomic sequencing of samples collected from a variety of ocean settings around the world shows high taxonomic variability (even at the phylum level) with relatively stable distribution of categories of functional genes [1], and that the environmental conditions predict the composition of the community in terms of functional groups better than in taxonomic structure, suggesting that functional and taxonomic structure may constitute roughly independent "axes of variation" in which functional structure captures most of the variation predicted by environmental conditions [2]. The same pattern of conserved functional community structure across variation in taxonomic structure is seen in the human microbiome [3-6], and in microbial communities assembled in vitro on a single nutrient resource [7]. Convergence of functional community structure with variation in species structure as a result of assembly history is also seen in plant communities [8], suggesting that explaining this pattern can have application beyond microbial ecology. A study of functional structure in in vitro community assembly [7] presents a mathematical model based on the MacArthur consumer-resource dynamics model, which numerically reproduces this pattern, but the model is not analyzed.

Here I present a general class of consumer-resource models that describes the community-wide abundances of functional traits together with the abundances of species, and analyze these models to explain how regularity of functional structure can be an outcome despite variability in species composition, and when this outcome can occur in communities governed by resource-consumer interactions. I have used these models to construct a series of simulation experiments applying this result to functional community structure across variation in enviromental conditions and in taxonomic community structure.

First I tested a scenario in which functional structure was preserved in a single community across changes 
in species abundances as its environment changes. Second I turned to the question of when multiple communities converge to a common functional structure despite differing taxonomic composition. I present mathematical analysis of when this result occurs, and then three model examples. In one example, functional structure coincided with high-level (genus or higher) taxonomic composition, and community structure at that level was conserved across multiple communities with different histories of assembly and different species composition. In the second, functional traits were shared across taxa and co-occurring in diverse compositions within organisms, so that functional structure was not reflected at a higher taxonomic level, and conservation of functional structure was achieved by a complex balance of functionally overlapping species. Third is a simulated controlled experiment in which selected traits were upregulated and downregulated by manipulation of the environment while other traits were unaffected, in a community model similar to the second, above.

\section{Trait abundances in consumer-resource models}

A standard model framework for resource-consumer dynamics is widely used and well understood, particularly given a finite number of distinct species without spatial patchiness [9-11].

Resource abundances are increased by supply from outside the model community, and decreased by uptake by consumer species, and species abundances are increased by reproduction at a rate that depends on resource consumption, and decreased by fixed per-capita mortality. For example, one such model has this form:

$$
\begin{aligned}
& \frac{d X_{i}}{d t}=\sum_{j} c_{i j} r_{i j} R_{j} X_{i}-m_{i} X_{i}, \quad i=1, \ldots, n_{s} \\
& \frac{d R_{j}}{d t}=s_{j}-\sum_{i} r_{i j} R_{j} X_{i}, \quad j=1, \ldots, n_{r},
\end{aligned}
$$

where $X_{i}$ is the abundance of consumer species $i$ and $R_{j}$ is the abundance of resource $j$, while $r_{i j}$ is the consumption rate of resource $j$ by consumer $i, c_{i j}$ is a conversion rate of resource $j$ into reproductive fitness of $i, m_{i}$ is the per-capita mortality rate of consumer $i$, and $s_{j}$ is the rate of supply of resource $j$.

To analyze the behavior of functional traits and genes across the community, it is necessary to include a definition of trait abundance in the model. Let us assume that a species that consumes a given resource has a trait of consumption of that resource. Thus given $n_{r}$ resources I define the corresponding $n_{r}$ traits, one for each, which each consumer may possess or not: let $A_{i j}$ be one if consumer $i$ has trait $j$ and zero if 
not, and let $f_{i j}(\mathbf{R})$, the functional response, or uptake rate of resource $j$ by consumer $i$, be a continuous, nondecreasing, nonnegative function of the vector $\mathbf{R}$ of resource abundances. Trait assignments $A_{i j}$ that take on a greater range of nonnegative values may also be of interest, for future research.

Including this description of trait possession, a consumer-resource dynamics model has the form

$$
\begin{aligned}
\frac{d X_{i}}{d t} & =\sum_{j} A_{i j} c_{i j} f_{i j}(\mathbf{R}) X_{i}-m_{i} X_{i}, \quad i=1, \ldots, n_{s} \\
\frac{d R_{j}}{d t} & =s_{j}-\sum_{i} A_{i j} f_{i j}(\mathbf{R}) X_{i}, \quad j=1, \ldots, n_{r} .
\end{aligned}
$$

A type I functional response has the linear form

$$
f_{i j}(\mathbf{R})=r_{i j} R_{j}
$$

and a type II functional response (e.g. [12]) can take at least two forms:

$$
f_{i j}(\mathbf{R})=\frac{r_{i j} R_{j}}{1+h_{i j} R_{j}}
$$

or

$$
f_{i j}(\mathbf{R})=\frac{r_{i j} R_{j}}{1+h_{i j} \sum_{k} A_{i k} R_{k}},
$$

depending on whether saturation occurs independently for each trait a consumer possesses, with $h_{i j}$ as a constant describing how quickly resource consumption saturates in response to its availability. The functional response may also be a type III response [13], which can be described by a variety of mathematical forms. In the example model systems I present below, I use the type I and the first of the above two type II functional response forms.

There are at least two measures of abundance that can be used, motivated by forms of next-generation sequencing in widespread use.

Using a measure of possession of genes, as seen in metagenomic sequencing processes based on DNA sequences, the community-wide abundance of trait $j$ is defined as the total value of $A_{i j}$ over all consumers:

$$
T_{j}=\sum_{i} A_{i j} X_{i}
$$

A measure of expression of traits, more like the data reported by metatranscriptomic sequencing processes such as RNA-Seq, describes not the presence of genetic sequences but the rates at which their functions are actively used:

$$
E_{j}=\sum_{i} A_{i j} X_{i} f_{i j}(\mathbf{R})
$$


This paper analyzes conditions under which trait abundances $T_{j}$ and $E_{j}$ remain unchanged or nearly so while species abundances $X_{i}$ vary, and when species composition, in the sense of the presence and absence of specific species in a community, varies across communities. I present conditions for conservation of both measures of traits across environmental conditions and community structures, and examples in which the abundances of genetic material $T_{j}$ are conserved, the more stringent case.

\subsection{Analysis of consumer-resource models}

Given the above form of model, the behavior of these models is well understood [9-11]. When the community consists of a single consumer species dependent on a single limiting resource, the population size grows until its increasing resource consumption lowers the resource abundance to a level at which the consumer's reproduction and mortality rates balance. In this way, the resource abundance is regulated by the consumer: the abundance of the resource at equilibrium is a quantity determined by those organisms' processes of reproduction and mortality.

The population brings its limiting resource to the same equilibrium level, conventionally known as $R^{*}$ [11], regardless of whether the flow of the resource into the community is small or large. If there is a large inflow, the population size grows until it is consuming the resource at an equally high rate, drawing the resource abundance down to the required level. If inflow is small, population size becomes as small as needed to balance the flows. In this way, the size of the population is determined by the resource supply rate, but the abundance of the resource is not.

When there are multiple species and multiple resources, for each species there are certain combinations of resource abundances that balance its birth and death rates. With $n_{s}$ species and $n_{r}$ resources, these equilibrium conditions take the form of $n_{s}$ equations, one for each population $X_{i}$, each in $n_{r}$ unknowns $R_{j}$ :

$$
\frac{d X_{i}}{d t}=\sum_{j} A_{i j} c_{i j} f_{i j}\left(\mathbf{R}^{*}\right) X_{i}^{*}-m_{i} X_{i}^{*}=0, \text { for each } i,
$$

where $\mathbf{R}^{*}$ is the vector $\left(R_{1}^{*}, \ldots, R_{n_{r}}^{*}\right)$ of equilibrium values of the resource abundances. Each of these equations, one for each $i$, can in principle be solved for the set of values of $R_{1}^{*}$ through $R_{n_{r}}^{*}$ that satisfy this condition. Note that these solutions are not affected by the population sizes $X_{i}^{*}$ as long as the population sizes are nonzero. The solution set of the $i$ th equation describes the set of values of the $n_{r}$ resources at which net growth of species $i$ is zero. The solution of all these equations simultaneously is the set of resource 
abundances at which all species' growth is at equilibrium. This is why $n_{r}$ resources can support at most $n_{r}$ coexisting populations in these models under most conditions: because outside of special cases, no more than $n_{r}$ equations can be solved for $n_{r}$ variables simultaneously $[9,10]$. The equilibrium resource abundances $R_{j}^{*}$, taken together, are the solution of that system of equations. Thus the combination of all $n_{r}$ resource abundances at equilibrium is determined by the requirements of all the consumer populations combined. Note that they are independent of the resource supply rates as well as of the consumer population sizes.

That balance of resources is enforced by the sizes of consumer populations: if resources increase above the levels that produce consumer equilibrium, consumer populations grow, drawing resources at increased rates, and the opposite if resource levels drop, until the resources are returned to the required levels and supply rates are matched by the rates of consumption. Equilibrium levels of consumers are determined not by the above equilibrium equations, but by the model's other set of equilibrium conditions:

$$
\frac{d R_{j}}{d t}=s_{j}-\sum_{i} A_{i j} f_{i j}(\mathbf{R}) X_{i}=0 .
$$

At equilibrium, the consumer population sizes must be whatever values $X_{i}^{*}$ are required to make the overall uptake rate of each resource $j$ described by this equation equal to the supply rate $s_{j}$, when the resources are at the levels $R^{*}$ implied by the earlier equilibrium conditions (4). Thus the consumer population sizes, all taken together, are determined by the supply rates of all the relevant resources taken together, given the equilibrium resource levels, in such a way that resource inflow and outflow rates are balanced. When each resource is controlled by multiple consumers all of whom use multiple resources, each consumer abundance is determined by all the resource supplies in balance with the other consumers in ways that may be difficult to predict or explain.

In summary, there is a duality of causal relationships between the two players in this system, resources and consumers, in which resource levels are determined by the consumers' physiology (4), and consumer levels are determined in a complex interlocking way by the resource supply rates given the above resource levels (5). 


\subsection{Conditions for conservation of trait abundances across differences in community composition}

Given an arbitrary assemblage of resource consumer species, described by some unconstrained assignment of values to the functions $f_{i j}$, mortality rates $m_{i}$, and conversion factors $c_{i j}$, without knowledge of those values nothing can be concluded about the abundances of species, resources, and traits that will be observed in the long term.

However, under certain constraints on the relationships between these values, it can be shown that trait abundances at equilibrium, given that enough consumer species coexist at equilibrium, are determined only by the resources' supply rates without dependence on the consumers' characteristics.

I have derived conditions for simple dependence of trait abundances on their resources' supply rates In the appendix (A.1), and I summarize them here.

Condition for conservation of rates of trait expression, E. The community-wide rate of expression of a trait, labeled $E_{j}$ above, is determined by the supply rate of its resource in all model communities of the above form, provided that the community is consuming all resources at equilibrium. This result is simply because $E_{j}$ is tied to the rate of resource uptake, which must match the supply rate of the resource at equilibrium.

The community-wide abundance of possession of a trait $\left(T_{j}\right)$ is not tied to supply rates in all cases, but conditions exist under which these abundances are directly predicted by supply rates independent of species abundances.

Simple condition for conservation of trait abundances, T. A condition for conservation of trait abundances $T_{j}$ is that there are constant numbers $k_{j}$, one for each resource $j$, for which

$$
\sum_{j} c_{i j} A_{i j} / k_{j}=m_{i}, \text { for each species } i
$$

If that condition is met, and the response functions $f_{i j}()$ are defined in such a way that there is a set of resource levels $R_{j}$ that can satisfy the constraint $f_{i j}(\mathbf{R})=1 / k_{j}$ for each $i$ and $j$ for which $A_{i j}>0$, at the same time, then those resource levels describe an equilibrium for each community structure, at which community-wide trait abundance $T_{j}$ will be held fixed at a level that depends only on the supply rate $s_{j}$, even though community structure and species abundances may vary.

This condition can be explained by recognizing that the resource uptake rates $f$ are a scaling factor 
between the raw trait abundances $T$ and the trait expression rates $E$ : since the expression rates are in a fixed relation to supply rates across communities, for the trait abundances to be fixed in that way as well, the ratio between the two, which is $f_{i j}$, must be fixed.

General condition for conservation of trait abundances, T. Condition (6), in which each trait abundance $T_{j}^{*}$ depends only on the supply rate of the one corresponding resource supply rate $s_{j}$, is a special case of a more general case in which the full vector of trait abundances is determined by the full vector of resource supply rates, the condition for which is the more abstract one that constants $K_{j k}$ exist for which

$$
\sum_{k} K_{j k} A_{i k} f_{i k}(\mathbf{R})=A_{i j}, \text { for each } i \text { and } j
$$

and

$$
\sum_{j} c_{i j} A_{i j} f_{i j}(\mathbf{R})=m_{i}, \text { for each } i
$$

simultaneously, for at least one value of $\mathbf{R}$.

Approximate conservation of trait structure. If either of these relations is not exactly but very nearly satisfied, then the model can almost exactly conserve the trait abundances. See (A.1) for more formal discussion of this point and derivation of the above conditions.

Simple construction of example models. Examples in this paper, below, are constructed using the simple condition (6) for conservation of trait abundances, by assigning all mortality rates equal to a constant value $m$, conversion rates $c_{i j}$ equal to a constant $c$, with a fixed number $p$ of traits assigned to each consumer. This satisfies (6) with $k_{j}=m / c p$ for each $j$.

Under these conditions, given that there is sufficient diversity within a community to fix resource abundances at the required equilibrium point, each trait $T_{j}$ will have equilibrium abundance $T_{j}^{*}=s_{j} / k_{j}$, independent of the trait assigments $A_{i j}$ and species abundances $X_{i}^{*}$. Species abundances are implied by the definition $T_{j}^{*}=\sum_{i} A_{i j} X_{i}^{*}$, and vary with the specifics of the community structure. The matter of sufficient diversity is closely related to the dimensionality of the space spanned by the rows of the matrix of values $A_{i j}$, so model examples are constructed requiring the $A$ matrices to be of full rank. 


\section{Example: Complex regulation of functional structure within a com- munity}

The above analysis implies that abundance of each of a palette of traits can be regulated by the availability of the one resource associated with that trait, even though every organism in the community possesses multiple such traits. I observed the regulation of community-wide trait abundances within a single community using a model of four resources and four consumers. For each resource I defined a trait corresponding to consumption of that resource, which was shared by multiple consumer populations. The first three resources were supplied at a constant rate, while the fourth resource was supplied at a rate that changed at discrete times. Species and trait abundances shifted in response to the changing supply of the fourth resource.

In this model, with type I functional responses as in (1), the trait assignments $A_{i j}$ were constructed such that each species consumed a different three of the four resources (Fig. 1A). The resource supply rate constants $s_{j}$ were held constant for resources 1,2 , and 3 , while $s_{4}$ was piecewise constant, changing between three different values at discrete moments (Figure 1B).

The abundances of the four consumer species making up the model community came to equilibrium when their habitat was unchanging, but when the supply rate of resource 4 changed, they all shifted to different equilibrium levels (Figure 1C). However, despite these complex shifts in all the consumer species' abundances, the community-wide abundances of the traits of consumption of the first three resources were conserved at equilibrium across these changes in community structure, aside from brief transient adjustments (Figure 1D). Of the four traits modeled, only the fourth changed in equilibrium abundance in response to the changing resource supply.

The community was able to regulate the community-wide abundance of the trait involved in consumption of the fourth resource independent of the other three consumption traits, despite that fact that multiple of the four traits coexisted in every organism in the community.

This model community achieved equilibrium by bringing trait abundances to the needed levels after each change in community structure, even though the sizes of the four populations embodying those trait abundances were all different after each change. The population sizes were all altered in just the way necessary to adjust the total abundance of the trait of consumption of resource 4 to match the changing supply rate of resource 4 and leave the other three unaltered. 
A.

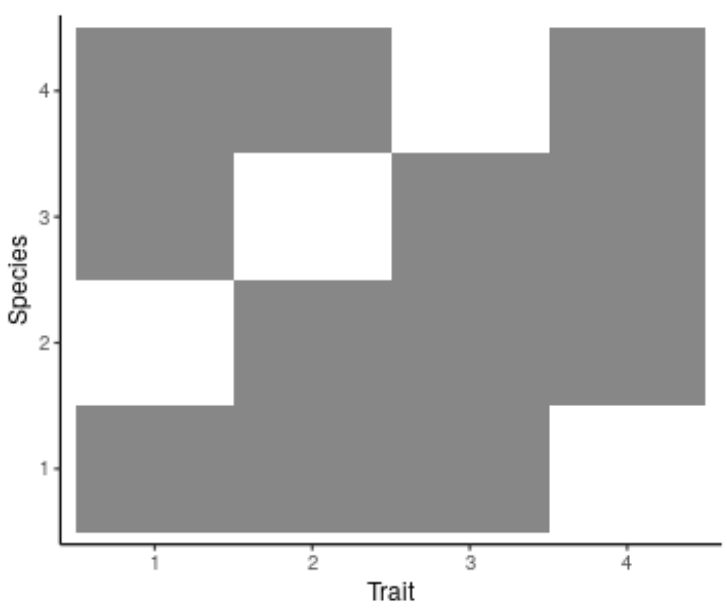

C.

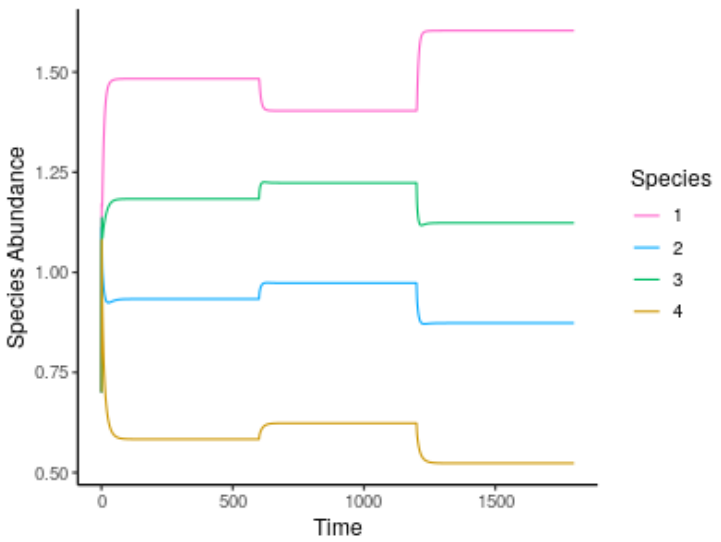

B.

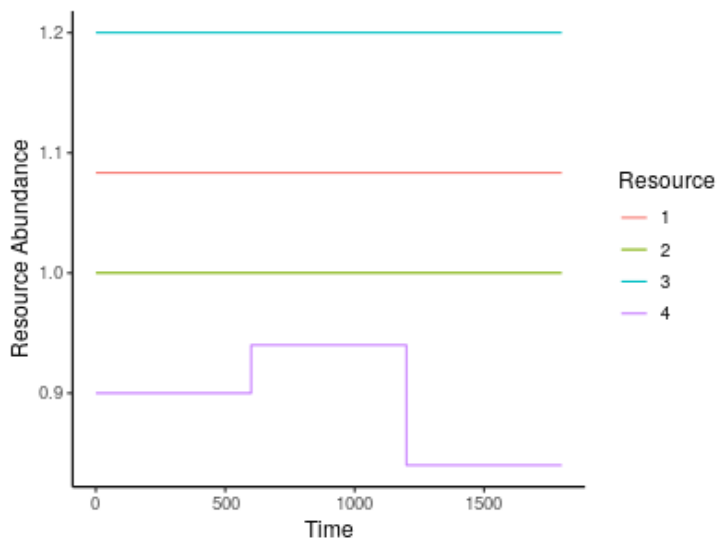

D.

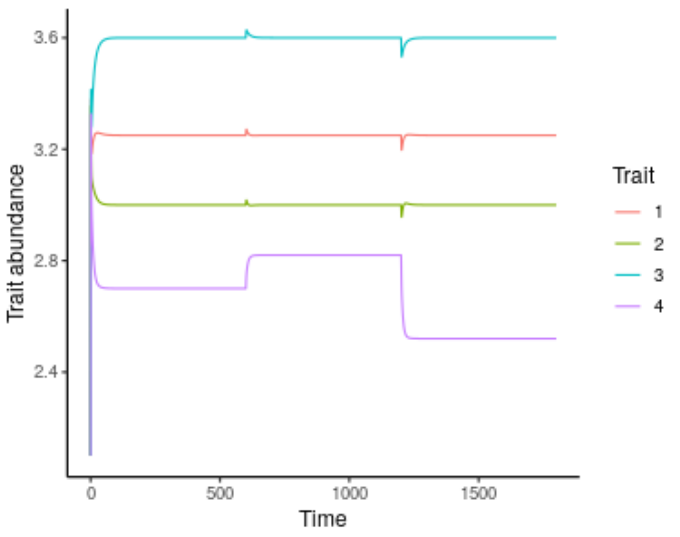

Figure 1: Regulation of functional structure within a community. A community model that satisfies the condition (6) maintains trait abundances fixed through changing environmental conditions by rebalancing all consumer population sizes. A. Assignment of traits to species $($ dark $=$ present, white $=$ absent); B. Supply rates of resources, with resources 1 through 3 supplied at constant rate, supply of resource 4 changing at discrete times. C. Species abundances all vary with changes in supply of resource 4 , while D. Wholecommunity trait abundances 1 through 3 are constant apart from transient fluctuations with only trait 4 changing in response to changing supply of resource 4.

\section{Example: Conservation of functional groups across differences in species composition}

One possible way in which communities may have a functional regularity that is not captured at the species level is that species may be interchangeable within guilds or groups of functionally equivalent species, where total counts in a group are conserved while species composition is not. The species in a group may be members of a family or phylum, or may be unrelated but perform similar functions. I constructed a model 
involving multiple guilds, in which members of each guild shared a functional trait of consumption of a guild-defining resource and varied in other traits. Communities were assembled by drawing species from a common pool of species.

Consumer species were grouped into three guilds, each guild defined by consumption of a guild-specific resource, with each species belonging to exactly one guild. Each species also consumed three other resources, assigned randomly from a common pool of five resources without regard to guild membership. Consumers' functional response to resources was type II (2). For parsimony, resource supply rates were set equal at a numerical value of $3 / 2$, and the saturation parameters $h_{i j}$, ideal uptake rates $r_{i j}$, conversion factors $c_{i j}$, and mortality rates $m_{i}$ were all set to 1 .

Thirty species were constructed, ten in each guild, by assigning non-guild traits at random conditional on the species-trait assignment matrix having the maximum possible rank $^{1}$, and thirty communities were constructed by randomly assigning twenty-one species to each, conditional on each community's $A$ matrix having maximal rank (Figure $2 \mathrm{~A}$ and $\mathrm{B})$.

The dynamics of these model communities was evaluated, starting from initial conditions at which all species and resource abundances were 1.0 in their respective units, for 200 time steps. Total abundances in each guild at the end of that time were plotted for comparison across communities.

At the end of that process, species composition varied across communities (Figure 2C), but the overall abundance of each guild was uniform across the model communities (Figure 2D).

\section{Example: Conservation of overlapping functional traits across differ- ences in species composition}

While in the above model, each species belonged to a single functional guild, I constructed a second model in which each consumer possessed multiple functional traits that were shared by other consumer species in varying combinations, so that regulation of trait abundances required a complex balancing of all the overlapping species.

The model was the same as above with the difference that rather than assigning species to guilds characterized by special traits, each species was assigned two of the ten resource consumption traits at random

\footnotetext{
${ }^{1}$ One fewer than the number of traits, or seven, is the highest rank this matrix can attain, given the requirement that guild traits sum to one and all traits sum to four for every species.
} 
A.

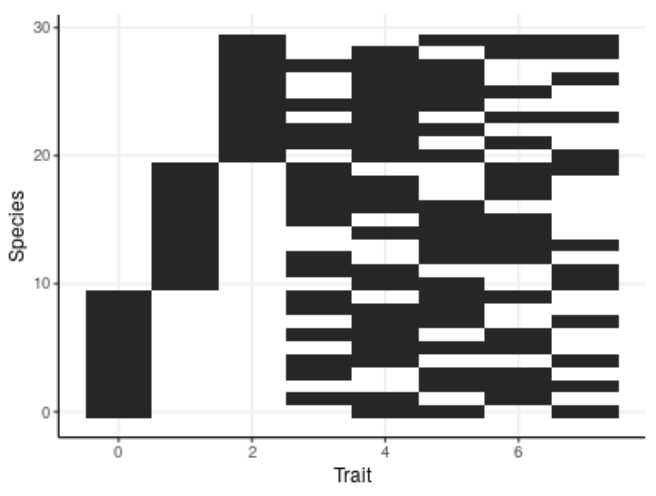

C.

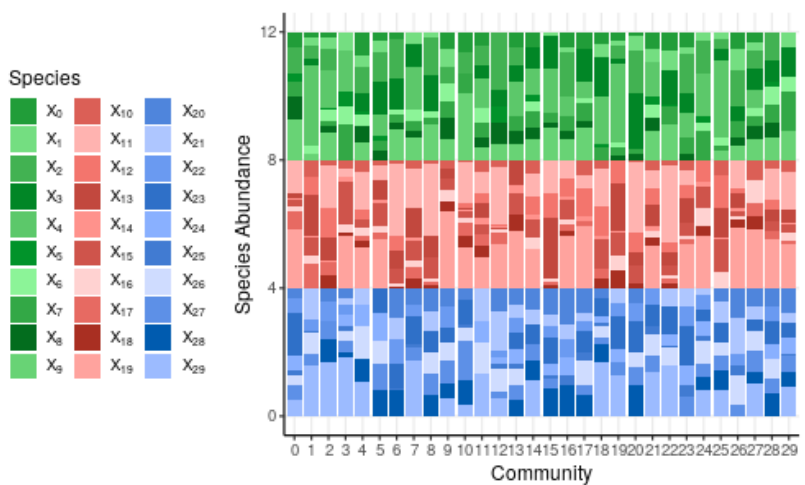

B.

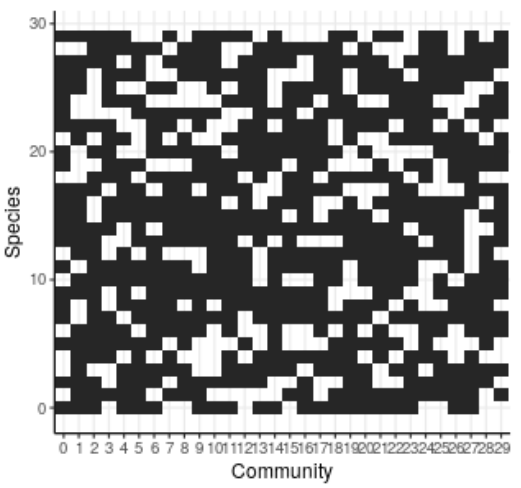

D.

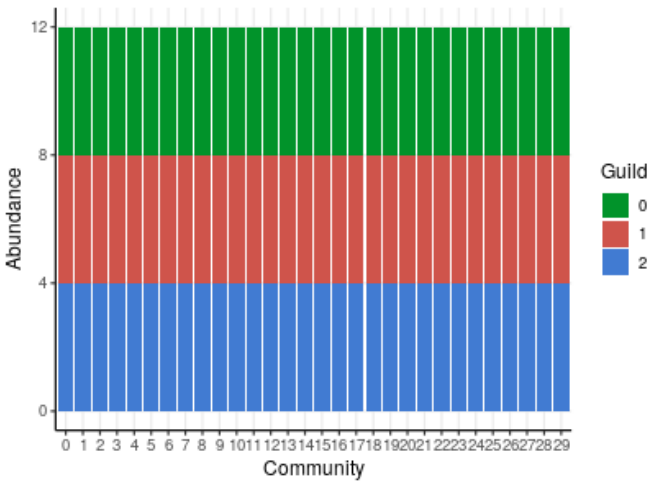

Figure 2: Conservation of functional groups across differences in species composition. Overall abundances of each of three "guilds" of consumers of different resources are held fixed across communities assembled randomly from varying species of each guild. (A.) Assignment of traits to species in guild model. Guild membership is defined by the first three traits, corresponding to consumption of "guild-defining resources," while the other five traits are guild-independent traits that distinguish species from one another. (B.) Assignment of species to communities. Some species assigned to communities may not survive beyond an initial transient as community comes to equilibrium. (C.) Species abundances (color coded by guild membership) and (D.) overall abundances of guilds at equilibrium, by community, in guild model. 
A.

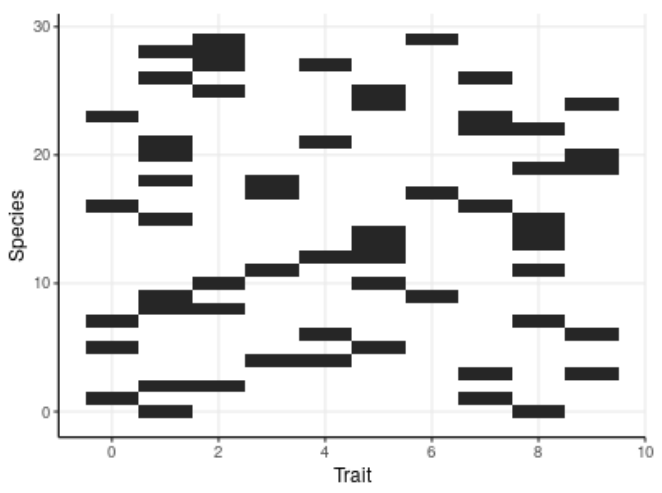

C.

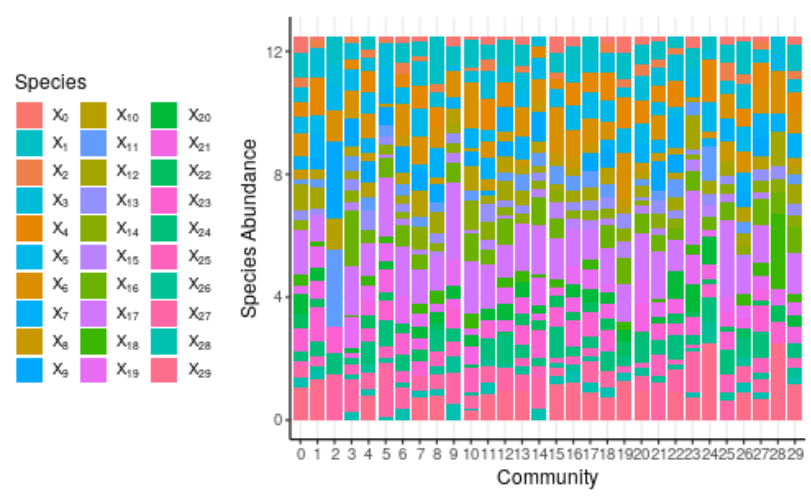

B.

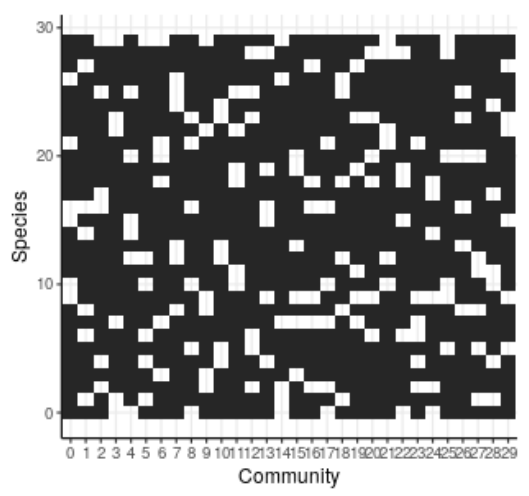

D.

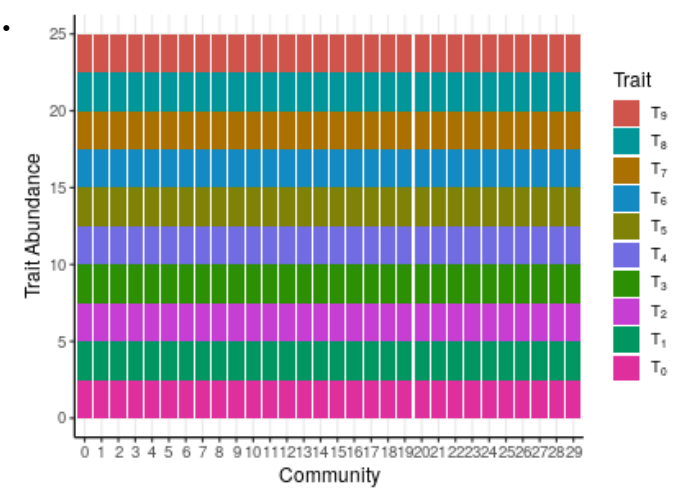

Figure 3: Conservation of overlapping functional traits across differences in species composition. When all consumption traits are randomly assorted across consumers, overall trait abundances are equal, as predicted by equal resource supply rates, independent of consumer species presence/absence or abundances. (A.) Assignment of traits to species, (B.) assignment of species to communities, (C.) species abundances at equilibrium, by community, and (D.) trait abundances at equilibrium, by community, in overlapping-traits model. Some species assigned to communities may not survive beyond an initial transient as community comes to equilibrium.

(Figure 3A). As above, I constructed 30 communities by assigning 24 species to each, chosen at random from a common pool of 30 candidate species, conditional on full rank (Figure 3B). Numerical parameters and functional responses were as in the above guild model, except that here $m_{i}=6.4, c_{i j}=4$, and $s_{i}=2$ for all $i$ and $j$. I recorded functional and taxonomic abundances after 200 time steps from initial conditions of uniform resource and species abundances of 1.0. At the end of that time I found that species abundances varied widely from community to community, but trait abundances were uniform across communities (Figure 3). 


\section{Example: Coexistence of conserved and variable traits}

Where the above model results explored cases in which functional community structure was the same across communities due to an underlying equality in conditions, here I look at how differences in conditions can be reflected by predictable differences in functional structure. The model I present here was constructed in the same way as in Section 5, with the difference that 15 communities were constructed and then evaluated subject to two different environments, labeled control and treatment. All parameters were set as above in the control arm of the experiment, while in the treatment arm the resources were partitioned into three classes, one whose supply rates were unchanged at 2.0, one in which supply rates were elevated to 2.8 , and one which which they were reduced to 1.2 .

Trait abundances at equilibrium (Figure 4D) clearly distinguished treated from control communities, and treated from unmodified resources. The traits associated with fixed-supply resources behaved like a "core functional structure" across these communities, while the traits associated with treated resources were variable in their abundances in accordance with the variation in resource supply. Species abundances varied between communities in both control and treatment groups (Figure 4C), and did not provide a visually apparent indicator of group membership.

\section{Discussion}

The above analysis has demonstrated, in a broad class of widely used models of consumer-resource ecological community dynamics, conditions on consumer physiology under which the community-wide abundance of traits, pathways, or genes involved in resource usage can be predicted by resource availability, independent of the taxonomic makeup of the community and the abundances of the taxa it includes.

In these model examples, and in general, the total rate of uptake of a given resource must balance the resource's net rate of supply. Therefore if the supply does not change, and the community continues to consume that resource, the uptake rate must return to the same level, after a possible transient fluctuation, after a perturbation in the community, and two communities comprised of different species but encountering the same rates of supply must necessarily manifest the same uptake rates.

In the models analyzed here, this matching of outflow to inflow can cause the community-wide rate of expression of traits associated with resource consumption to be conserved. If expression of a given trait 
A.

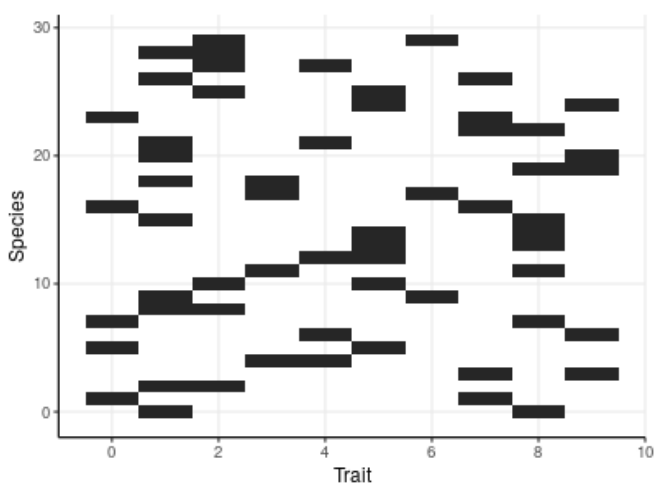

C.

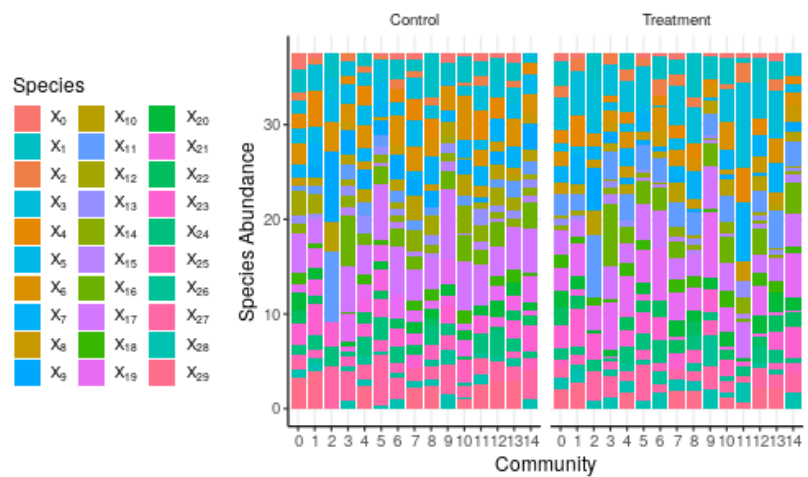

B.

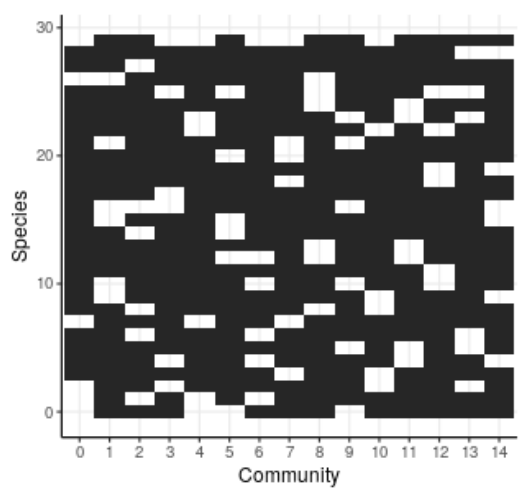

D.

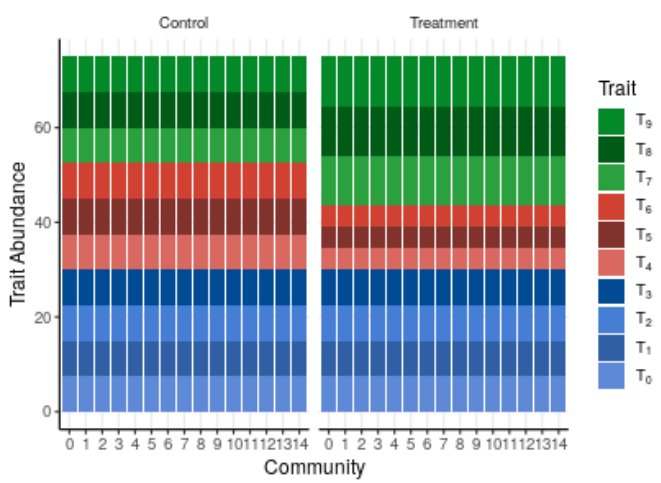

Figure 4: Coexistence of conserved and variable traits in simulated experimental conditions. Randomly assembled model communities are evaluated in "control" conditions of equal resource supply rates, and "treatment" conditions with altered supply rates. Trait abundances track supply rates across differences in community composition and across arms of the experiment. (A.) Assignment of traits to species, (B.) assignment of species to communities, (C.) Species abundances at equilibrium, by community and treatment arm, and (D.) trait abundances at equilibrium, by community and treatment arm, in simulated experiment model. Each community is simulated under both treatment and control conditions. Some species assigned to communities may not survive beyond an initial transient as community comes to equilibrium. 
or pathway is directly related to the rate of consumption of a resource, it is natural that the rate of trait expression should be predicted by the rate of resource supply because of the relation between supply rate and overall uptake rate. Even though those traits may be distributed across multiple consumer species, each involved with multiple resources, a central result of these models is that species abundances are driven by resource availability in such a way as to regulate all the resources simultaneously [10], even if that balance requires a complex adjustment of all the species in the community. In this way, community-wide rates of trait expression can be matched to resource supplies even though all species abundances may vary irregularly in whatever ways are needed to make uptake rates balance supply rates.

Such a concept of trait expression (the quantity $E_{j}$ defined above) is likely more appropriate to a metatranscriptomic description of a community, as implemented by high-throughput sequencing techniques such as RNA-Seq [14], than to a metagenomic description. A metagenomic description generated by measuring abundances of DNA sequences in cells may be better described as a measure of trait or gene prevalence, in the sense of abundance of organisms possessing the genes, such as the quantity $T_{j}$ used here. This quantity can also be tied to resource supply rates as trait expression can, but the relation is not as universal and requires more conditions. In summary, organisms that possess a genetic pathway may use it at varying rates depending on the availability of the resources involved, and on whether conditions are more favorable to the use of other pathways. In terms of the model dynamics, the uptake rate depends both on the abundance of organisms possessing the relevant trait and on the availability of the various resources relevant to those organisms. The conditions for conservation of the overall prevalence of such genes across the community include regulation of resource uptake rates per capita to common levels across communities. In many if not all conditions, this likely requires control of resource abundances to common levels across communities (the vector $\mathbf{R}^{*}$ ). Under those conditions, a fixed relation between trait expression and trait prevalence is maintained, allowing both to be conserved across differences in consumer species.

The examples in this article have demonstrated this more stringent condition of regulation of trait prevalences, for illustration purposes. They showed a series of different results involving regulation of community functional structure, as defined by trait prevalences, that can be manifest by this effect.

The first example demonstrated that in a single model community, a temporal fluctuation in one resource supply rate can induce a coordinated shift in all of the species abundances, though its effect on the trait abundances is restricted to the one trait, leaving the community's functional structure otherwise unchanged. 
Second, in a model of guilds of consumers specialized on different resources, that is, each characterized by a different resource-consumption trait, the overall size of each guild was shown to be predicted directly by resource supply, across multiple community structures, while the species composition of each guild varied widely across communities. This follows from the definition of guilds which makes their sizes effectively identical to trait prevalences.

Next was a model in which consumption traits were not partitioned into disjoint guilds, but shared in overlapping ways by consumers of multiple resources. In multiple model communities differing widely in species composition, the community-wide abundance of consumption traits was nonetheless seen to be uniform across communities when resource supplies were uniform.

Finally, differences in resource supply in a model controlled experiment were shown to produce regular, predictable differences in trait prevalences across model communities while core functional traits corresponding to unaltered resources were held fixed, at the same time that all species abundances varied across communities and treatment groups in apparently irregular ways.

All the above examples demonstrated conditions in which functional structure of a community is exactly determined by its environment, independent of its taxonomic composition. The theory that predicts these outcomes also predicts that functional structure will be approximately conserved across community structures if the conditions are nearly enough met, an important consideration when attempting to apply such results to the imprecise world of biology outside of models.

These results demonstrate a mechanism by which functional structure can be predicted directly from environmental conditions in a simple case, bypassing the complexities of taxonomic variation. It should be read not as a faithful model to be applied directly to communities in the lab or field, but as a step toward a fuller theory to describe them. This paper offers a proof of concept that conservation of trait abundances can be explained by known models of community dynamics, and that functional observations of communities can describe and predict their behavior more parsimoniously than taxonomic observations.

Interestingly, these results turn out to be insensitive to the range of different types of functional response curves that can be manifested by resource consumers. Instead, they require a condition of uniformity of response across consumer taxa. The different consumers' responses to resource availability must satisfy a somewhat opaque consistency condition, to allow the total presence of resource consumption traits to be held in a consistent relation to resource supply at the same time that the rates of trait expression are as 
well. In the example model results presented above, this is achieved by assuming the functional response to each resource is the same among all consumers that use it, which is an especially simple way to satisfy the condition, regardless of the type of functional response. Note that the result does not require that all species included in community assembly meet these conditions, but only that they be met by the consumers that are present in the community at equilibrium.

This work has multiple limitations. It does not apply directly to communities whose dynamics are shaped by interactions other than resource competition, for example bacteria-phage interactions, direct competition or facilitation between microbes, or host-guest interactions such as host immunity. While similar results may hold in these cases, they require expanded models to investigate them. The assumptions made here about the close mapping between trait expression and uptake rates are likely not satisfied in many cases, and should be unpacked to allow a fuller treatment of the subject. Spatial heterogeneity alters the behavior of consumerresource models and must be studied separately, and can open up additional interesting questions such as the response of communities to spatially variable resource supply. Endogenous taxonomic heterogeneity driven by local dispersal may not imply comparable functional heterogeneity if underlying abiotic conditions are homogeneous. The analysis of equilibrium community structures also likely does not apply to many communities, and it may be worthwhile to expand the analysis to describe slow dynamics of community structure in conditions of constant immigration, seasonal or other temporal variability in the environment, or evolutionary change in which equilibrium is not attained.

It is not obvious whether the conditions presented here for compatibility of vital rates across taxa to make trait abundances behave regularly are realistic for microbes. Having established that these are the necessary conditions in these models, if they are not considered believable, then this work serves to illuminate the questions that must be answered about how microbial communities diverge from these models, and how else their observed functional regularities can be explained. One avenue might be to investigate whether $R^{*}$ competition under conditions of high diversity can reduce a community without the closely matched $R^{*}$ conditions described here to a subcommunity in which such a condition is roughly though not precisely met.

These results suggest a number of further questions to be investigated, such as the impact of more complex mappings between genetic pathways and resource uptake dynamics, and the dynamics of functional community structure in the presence of mechanisms such as direct microbe-microbe interactions or host immunity. The dynamics of traits involved in functions other than resource consumption is left to be 
studied, such as for example drug resistance or dispersal ability, as is the impact of evolutionary dynamics, including horizontal transfer, on the dynamics of functional composition. It may be productive to investigate whether a community's need to regulate its functional composition in certain ways can lead to selection for certain kinds of genetic robustness, dispersal, horizontal transfer, or other characteristics. It would be of interest to study whether conditions selecting for sharing of traits across taxa can be distinguished from those selecting for specialization by taxon. The present study is offered as an initial investigation, presenting an existence proof of the ability of a community to regulate its functional composition independent of its taxonomic makeup, in hope it will open doors to further work.

Community ecology theory often focuses on questions of import primarily to communities of plants and animals, examining models of interactions among a relatively small number of species, whose traits are stably defined, to explain patterns of coexistence and diversity. In microbial ecology, where organisms of different taxa share and exchange genes, and communities can be very diverse and variable in composition over time and space, theoretical questions particular to microbial ecology may be posed, potentially driving ecological theory into new and productive arenas.

\section{Acknowledgements}

This study was partially supported by a Models of Infectious Disease Agent Study (MIDAS) grant from the US NIH/NIGMS to the University of California, San Francisco (U01GM087728). LW is grateful to Peter Ralph for a comment that motivated this project, and to PR, Todd Parsons, Sue Lynch, Travis Porco, Sarah Ackley, Rae Wannier, and several anonymous reviewers for helpful conversation and feedback.

\section{References}

[1] Sunagawa S, Coelho LP, Chaffron S, Kultima JR, Labadie K, Salazar G, et al. Structure and function of the global ocean microbiome. Science. 2015 May;348(6237):1261359. Available from: http://science. sciencemag. org/content/348/6237/1261359.

[2] Louca S, Jacques SMS, Pires APF, Leal JS, Srivastava DS, Parfrey LW, et al. High taxonomic variability despite stable functional structure across microbial communities. Nature Ecology \& Evolution. 2017 Jan;1(1):0015. Available from: https://www.nature.com/articles/s41559-016-0015. 
[3] Turnbaugh PJ, Hamady M, Yatsunenko T, Cantarel BL, Duncan A, Ley RE, et al. A core gut microbiome in obese and lean twins. Nature. 2009 Jan;457(7228):480-484. Available from: http: //www.nature.com/nature/journal/v457/n7228/full/nature07540.html.

[4] The Human Microbiome Project Consortium. Structure, function and diversity of the healthy human microbiome. Nature. 2012 Jun;486(7402):207-214. Available from: https://www.nature.com/nature/ journal/v486/n7402/abs/nature11234.html.

[5] Gosalbes MJ, Abellan JJ, Durbán A, Pérez-Cobas AE, Latorre A, Moya A. Metagenomics of human microbiome: beyond 16s rDNA. Clinical Microbiology and Infection. 2012 Jul;18:47-49. Available from: http://onlinelibrary.wiley.com/doi/10.1111/j.1469-0691.2012.03865.x/abstract.

[6] Gosalbes MJ, Durbán A, Pignatelli M, Abellan JJ, Jiménez-Hernández N, Pérez-Cobas AE, et al. Metatranscriptomic Approach to Analyze the Functional Human Gut Microbiota. PLOS ONE. 2011 Mar;6(3):e17447. Available from: http://journals.plos.org/plosone/article?id=10.1371/ journal.pone.0017447.

[7] Goldford JE, Lu N, Baji D, Estrela S, Tikhonov M, Sanchez-Gorostiaga A, et al. Emergent simplicity in microbial community assembly. Science. 2018 Aug;361(6401):469-474. Available from: http:// science. sciencemag. org/content/361/6401/469.

[8] Fukami T, Martijn Bezemer T, Mortimer SR, van der Putten WH. Species divergence and trait convergence in experimental plant community assembly. Ecology Letters. 2005;8(12):1283-1290. Available from: http://dx.doi.org/10.1111/j.1461-0248.2005.00829.x.

[9] MacArthur R, Levins R. Competition, Habitat Selection, and Character Displacement in a Patchy Environment. Proceedings of the National Academy of Sciences of the United States of America. 1964;51(6):1207-1210. Available from: http://www.jstor.org/stable/72141.

[10] Levin SA. Community Equilibria and Stability, and an Extension of the Competitive Exclusion Principle. The American Naturalist. 1970 Sep;104(939):413-423. Available from: http://www.journals . uchicago.edu/doi/10.1086/282676.

[11] Tilman D. Resource Competition and Community Structure. Princeton University Press; 1982. 
[12] Holling CS. Some characteristics of simple types of predation and parasitism. The Canadian Entomologist. 1959;91(7):385-398.

[13] Holling CS. The components of predation as revealed by a study of small-mammal predation of the European pine sawfly. The Canadian Entomologist. 1959;91(5):293-320.

[14] Wang Z, Gerstein M, Snyder M. RNA-Seq: a revolutionary tool for transcriptomics. Nature reviews genetics. 2009;10(1):57.

\section{A Appendix}

\section{A.1 Analysis of conditions for conservation of trait structure across composition}

This section presents a mathematical analysis of the conditions under which the model presented above conserves community functional structure across variation in the selection of species composing the community.

Let us assume $n_{r}$ resources, and a community defined by $n_{s}$ species coexisting at equilibrium, parametrized by constants $A_{i j}, c_{i j}$, and $m_{i}$, and the functions $f_{i j}(\mathbf{R})$. We wish to analyze conditions for the vectors of trait abundances $\mathbf{T}$ and $\mathbf{E}$ at equilibrium to be independent of the community composition. That is, imagine a large pool of species described by $A, c$, and $m$ values and response functions $f$ : how must those values be constrained such that when a community is assembled from a subset of those species, the resulting trait abundances at equilibrium are the same regardless of which assemblage of those species was chosen.

This section will analyze the model equations in matrix form. A model community is parametrized by constant matrices $\mathbf{A}$ and $\mathbf{c}$ and vectors $\mathbf{m}$ and $\mathbf{s}$, and a matrix $\mathbf{f}$ whose entries are functions $f_{i j}$ of the resource abundances $R_{j}$. The state of the model system is vectors $\mathbf{X}$ and $\mathbf{R}$, and derived vectors $\mathbf{T}$ and $\mathbf{E}$. Many of these objects have equilibrium values $\mathbf{X}^{*}, \mathbf{R}^{*}$, etc. $\mathbf{1}$ is a vector of ones. The operator $\odot$ stands for elementwise multiplication.

The relevant relations are as follows. First there are the equilibrium conditions for the $X$ variables,

$$
(\mathbf{c} \odot \mathbf{A} \odot \mathbf{f}) \mathbf{1}=\mathbf{m},
$$

and for the $R$ variables,

$$
\mathbf{s}=(\mathbf{A} \odot \mathbf{f})^{T} \mathbf{X} .
$$

The trait abundances are defined in terms of the $X$ variables:

$$
\begin{aligned}
& \mathbf{T}=\mathbf{A}^{T} \mathbf{X} \\
& \mathbf{E}=(\mathbf{A} \odot \mathbf{f})^{T} \mathbf{X} .
\end{aligned}
$$


It follows immediately from the equilibrium condition (eq-R) that the equilibrium vector $\mathbf{E}^{*}$ of trait expression rates is entirely determined by the vector of supply rates $\mathbf{s}$, regardless of the population abundances $\mathbf{X}$ and per-consumer uptake rates $\mathbf{f}$ that are the components of the expression rates. Because these expression rates are defined equal to the rate of uptake of the resources, and equilibrium requires the rate of uptake to equal the rate of supply, no specific conditions on the structure of the community are needed to guarantee this result. For this reason, trait expression rates in this model are conserved across community structures. However, conditions for conservation of the vector $\mathbf{T}^{*}$ of rates of presence of traits are more restrictive and require more analysis.

The first equation (eq-X) is solved by finding values of $f_{i j}$ that bring the two sides to equality. As written this equation is underdetermined as there are $n_{s}$ conditions for $n_{s} n_{r}$ values $f_{i j}$. However, the $f$ variables are also constrained by their dependence on the $n_{r}$-dimensional vector $\mathbf{R}$. Because of that condition, the matrix f does not range freely over $n_{s} n_{r}$ dimensions, but over an $n_{r}$-dimensional submanifold of that space defined by the parametrization $\mathbf{f}(\mathbf{R})$ :

$$
\mathbf{f}=\mathbf{f}(\mathbf{R}) \text {. }
$$

An equilibrium matrix of resource uptake rates $\mathbf{f}^{*}$ is found by solving (eq-X) and (f-R) simultaneously. The functional forms of the response functions $f_{i j}(\mathbf{R})$ can be substituted into (eq-X) to yield a system of $n_{s}$ equations in the $n_{r}$ variables $R_{j}$. In the generic case, when $n_{s}=n_{r}$, this determines a unique solution vector $\mathbf{R}^{*}$, which determines the values of all entries of the matrix $\mathbf{f}^{*}=\mathbf{f}\left(\mathbf{R}^{*}\right)$. In other cases, multiple solutions for $\mathbf{R}^{*}$ and $\mathbf{f}^{*}$ may be possible.

Given $\mathbf{f}^{*}$, equilibrium population sizes are described by $($ eq- $R)$. If the matrix $\left(\mathbf{A} \odot \mathbf{f}^{*}\right)^{T}$ is square and singular, then the vector $\mathbf{X}^{*}$ of equilibrium population sizes is the unique solution of (eq- $\mathrm{R}$ ). If the matrix is nonsingular, then there can be a space of solutions for $\mathbf{X}^{*}$.

The trait abundances $\mathbf{T}^{*}$ must satisfy $(\mathrm{T})$ given equilibrium values of $\mathbf{X}$.

Now let us imagine that the community's equilibrium trait abundances can be predicted from the resource supply alone, without dependence on the parameters describing the species in the community. The above relations show that given a community structure, both $\mathbf{T}^{*}$ and $\mathbf{s}$ are linearly related to $\mathbf{X}^{*}$. For their relation to be independent of the community, let us assume

$$
\mathbf{T}^{*}=\mathbf{K} \mathbf{s}
$$

for some constant matrix $\mathbf{K}$.

This implies that

$$
\mathbf{T}^{*}=\mathbf{K}\left(\mathbf{A} \odot \mathbf{f}^{*}\right)^{T} \mathbf{X}^{*} .
$$


Comparing this to $(\mathrm{T})$, it can be satisfied if

$$
\mathbf{K}\left(\mathbf{A} \odot \mathbf{f}^{*}\right)^{T}=\mathbf{A}^{T}
$$

or

$$
\sum_{k} A_{i k} f_{i k}\left(\mathbf{R}^{*}\right) K_{j k}=A_{i j}
$$

for each $i$ and $j$.

Given a community parametrized by the constant matrix $\mathbf{A}$ and the functional forms $f_{i j}()$, the above equation describes a set of $n_{s} n_{r}$ constraints on the resource concentrations $R_{j}^{*}$ and trait assignments $A_{i j}$, which must be satisfied at equilibrium simultaneously with the previously discussed constraints.

The above solves a general case of the problem, in which the entire vector $\mathbf{T}^{*}$ of trait abundances is determined by the full vector $\mathbf{s}$ of supply rates. The more restrictive case that each trait abundance $T_{j}^{*}$ depends only on the supply of resource $j$, rather than on all the resources' supply rates, requires the matrix $\mathbf{K}$ to be diagonal. In this case, the condition becomes

$$
A_{i j} f_{i j}\left(\mathbf{R}^{*}\right) k_{j}=A_{i j}
$$

for each $i$ and $j$, where $k_{j}$ is the $j$ 'th diagonal entry of $\mathbf{K}$, or

$$
f_{i j}\left(\mathbf{R}^{*}\right)=1 / k_{j}
$$

for all $i$ and $j$ for which $A_{i j}$ is nonzero.

This condition implies that for each resource, the equilibrium uptake rates $f^{*}$ of that resource must be equal across its consumer species, and equal to a value that is uniform across different community structures.

Given that, the resource concentrations are those implied by these values of $f$, and the species abundances are a solution of (eq-R). Note that that species abundances can vary depending on A. Also, I note that this condition can permit more than $n_{r}$ species to coexist on $n_{r}$ resources, as it makes them compatible in a non-generic way.

Note also, however, that because the operations of pointwise multiplication and division, matrix multiplication, and matrix inversion are continuous in the values of all matrix entries, the above results have the property that if the above two conditions are nearly met, that is, if the $f$ and $A$ entries are within a suitably small distance $\varepsilon$ of values that satisfy the conditions exactly, then the trait abundances will be close to values that are exactly conserved. In other words, the functional regularity in question is approximately achieved when the conditions are nearly enough met. In this approximate but not exact case, the model does behave generically and its diversity can be expected to be limited by the number of resources. 
If resources have multiple consumers apiece, the result that for each resource $j, f_{i j}\left(\mathbf{R}^{*}\right)=1 / k_{j}$ across all consumers $i$ of resource $j$ does not require that the response function have a uniform form across consumers, $f_{i j}(\mathbf{R}) \equiv f_{j}\left(R_{j}\right)$, but that is certainly one way it can be achieved.

The example models in this paper are a special case of this condition, constructed by assigning some fixed number $p$ of traits to each species, and setting all consumers' functional response curves for each resource equal, with $m_{i} \equiv m$ and $c_{i j} \equiv c$. In this case, (eq-X) is satisfied by $f_{i j}^{*}=m / p c$ for all $i$ and $j$, which also satisfies condition (7) with $k_{j}=p c / m$. Equilibrium resource abundances are $R_{j}^{*}=f_{i j}^{-1}(m / p c)$ for each $j$, for any consumer $i$, which is well-defined given that the functions $f_{i j}()$ are assumed independent of $i$. Under these assumptions, the above results imply that $T_{j}^{*}=p c s_{j} / m$ for each $j$. 